\title{
Effect of Common Bean Consumption on the Gut Associated Microbiome in an In Vivo Screening Model for Breast Cancer ${ }^{+}$
}

\author{
Henry J. Thompson ${ }^{1, *}$, John N. McGinley ${ }^{1}$, Elizabeth S. Neil ${ }^{1}$ and Tiffany L. Weir ${ }^{2}$ \\ 1 Cancer Prevention Laboratory, Colorado State University, Fort Collins, CO 80523, USA; \\ john.mcginley@colostate.edu (J.N.M.); elizabeth.neil@colostate.edu (E.S.N.) \\ 2 Department of Food Science and Human Nutrition, Colorado State University, Fort Collins, CO 80523, \\ USA; tiffany.weir@colostate.edu \\ * Correspondence: henry.thompson@colostate.edu; Tel.: +1-970-491-7748; Fax: +1-970-491-3542 \\ + Presented at the The 1st International Electronic Conference on Nutrients - Nutritional and Microbiota \\ Effects on Chronic Disease, 2-15 November 2020; Available online: https://iecn2020.sciforum.net/.
}

Published: 30 October 2020

\begin{abstract}
Population data indicate that consumption of common bean reduces breast cancer risk, an effect replicated in a well characterized rodent model of mammary carcinogenesis. However, low molecular weight fractions of bean failed to inhibit growth of established breast cancer cell lines. Given that cell culture screens for anticancer activity are designed to detect a decrease in cell number accumulation, we reasoned that it might be possible to create a cell number accumulation assay for screening foods such as common bean in vivo by using an oncogene driven model for breast cancer. A benefit of this approach is that it permits simultaneous detection of systemic effects in the host and their potential mediation by the gut microbiome. We report an in vivo mammary cell accumulation assay driven by the polyoma middle $\mathrm{T}$ antigen (PyMT) oncogene and show that bean feeding reduced the accumulation of cells in developing mammary pathologies. As a candidate mediator, we report the impact of bean consumption on the gut associated microbiome. Differences were observed between common bean and the control diet in microbial phylogenetic diversity, beta diversity, abundance of various taxa, and predicted functional activity.
\end{abstract}

Keywords: common bean; gut microbiome; metagenomics; mammary carcinogenesis; rapid screening model

\section{Introduction}

There is a growing appreciation of food as a valuable "unit of reduction" in advancing our understanding of the effective use of the dietary component of lifestyle in the prevention and control of cancer [1]. People eat foods, not nutrients and phytochemicals, and assessment of patterns of food consumption, also referred to as dietary patterns, is providing useful insights across a range of chronic diseases [2]. However, foods and food patterns are complex, and there is an inherent interest within the scientific community to identify the origins of protective activity, particularly with respect to the interaction of food(s) with the gut associated microbiome [3-6]. The work reported herein illustrates a strategy for mechanistic inquiry about the effect(s) of foods and food patterns on the gut microbiome in a screening model for breast cancer specifically developed for this purpose.

A reasonable approach for the use of preclinical models to advance the field of diet and cancer research is to deconstruct observations made in either studies of populations or those resulting from clinical investigations. Thus, our laboratory noted that data from the Nurses' Health Study 1 implicated dietary intake of common bean and lentil as being associated with reduced risk for breast 
cancer in agreement with a second report in the Four Corners Study, a cohort with a different ethnic profile $[7,8]$. Accordingly, a well characterized rodent model for breast cancer was used to determine whether the cohort-based observations could be replicated, and the protective effect was shown to be common bean dose dependent $[9,10]$. With these findings in hand, the decision was made to interrogate the low molecular weight extracts of bean to determine if the antiproliferative and/or pro apoptotic activity observed in mammary carcinoma of bean fed rats could be replicated by treating established breast cancer cell lines with these extracts. The rationale for doing this was strengthened by our reported observation that low molecule fractions of bean were active in a longevity extension assay in C. elegans, an assay that involves incubation of the plant extract with a bacterium (E. coli.) on which the nematodes feed [11]. However, when the same fractions were evaluated for growth inhibitory activity against either the steroid hormone receptor positive (MCF-7) or steroid receptor negative human breast cancer cell line (MDMBA-232), no inhibition was observed. Given that at the rudimentary level of screening for anticancer activity in cell culture assays, one is simply evaluating for either an antiproliferative and/or proapoptotic effect that decreases cell number, we reasoned that it might be possible to create a cell number accumulation assay for screening foods for inhibitory activity in vivo by using an oncogene driven model for breast cancer. After establishing and characterizing the breast cancer screening model and determining that common bean consumption had an inhibitory effect on accumulation of cells that were diagnosed as mammary carcinoma, we determined whether differences could be detected in the gut associated microbiome that potentially could relate to effects on cell signaling pathways assessed in the in vivo screen. Both the screening model for cancer and the impact of bean consumption on the gut associated microbiome are reported herein.

\section{Materials and Methods}

\subsection{Animals and Genotyping}

C57BL/6J wild type mouse breeder pairs were obtained at 3-4 weeks of age (The Jackson Laboratory, Bar Harbor, ME, USA, JAX stock \#000664). The colony was maintained in our laboratory by mating wild-type males and females. B6.FVB-Tg(MMTV-PyVT)634Mul/LellJ male hemizygous mice were obtained from The Jackson Laboratory (JAX stock \#022974). These mice express the polyoma virus middle T antigen (PyMT) under control of the mouse mammary tumor virus (MMTV) promoter/enhancer, which induces the rapid formation of mammary tumors. As hemizygous females demonstrate loss of lactational ability, the colony was maintained by mating wild-type C57BL/6JB6 females with hemizygous PyMT males. Hemizygous PyMT female offspring are identified as described in $[12,13]$.

\subsection{Screening Assay}

Female mice of the correct genotype were used for this experiment. Following genotyping, hemizygous PyMT female mice were randomized to the treatment groups and initiated on experimental diets at 19-22 days of age. The formulation of the control and common bean diet are described in [14]. Two weeks following initiation of the diet, mice were euthanized, tissue harvested, and mammary gland whole mounts prepared and evaluated as previously described [15].

\subsection{Histological Assessment}

Following analysis of mammary gland whole mounts for lesion area, tissue was excised, reprocessed, and embedded in paraffin. Sections were cut at $5 \mu \mathrm{M}$, stained with hematotoxin and eosin and histopathologically classified according to previously published criteria [16].

\subsection{Western Blot-Based Immuno-Nanocapillary Electrophoresis}

Samples were prepared and assayed according to a protocol previously published by our laboratory [17]. The lysate supernatant was divided into $25 \mu \mathrm{L}$ single use aliquots using $0.2 \mathrm{~mL}$ PCR 
tubes and stored at $-80^{\circ} \mathrm{C}$. Protein concentration was determined using the Bradford assay. Nano capillary electrophoresis was performed using the WES instrument and proprietary kits (ProteinSimple, San Jose, CA, USA). Samples were denatured in a dry bath at $95{ }^{\circ} \mathrm{C}$ for $5 \mathrm{~min}$. Samples, biotinylated ladder, multiplexed primary antibodies, HRP-conjugated secondary antibody, chemiluminescent substrate (luminol-S peroxide) and wash buffer were pipetted into the appropriate wells according to kit instructions. The capillary cartridge and microplate were loaded into the fully automated WES instrument. The entire assay was completed within each capillary as follows: the vacuum manifold loaded each capillary with a separation matrix, stacking matrix and sample; a voltage of 375 volts was applied for $30 \mathrm{~min}$ to separate the proteins based on size followed by exposure to UV light in order to immobilize the proteins in the capillary prior to immuno-labeling and subsequent detection resulting in the chemiluminescent signal intensity of each target protein displayed as an electropherogram. Each sample capillary was probed with the primary antibody for the protein of interest and with an antibody for either beta actin or lamin B1 which were used as a loading controls depending upon which primary antibodies were multiplexed. All values reported are normalized to this loading control.

\subsection{Microbiota Characterization}

Using intestinal specimens collected at necropsy, DNA was extracted using the QIAamp PowerFecal DNA Kit (Qiagen, Germantown, MD, USA). $2 \times 250$ bp paired-end sequencing libraries of the V4 region of the 16s rRNA gene were constructed by using the Schloss MiSeq Wet Lab SOP followed by sequencing on an Illumina MiSeq instrument (Illuminia, San Diego, CA, USA) [18]. All $16 \mathrm{~S}$ rRNA gene sequences were demultiplexed and processed with the open source bioinformatics tool QIIME 2, version qiime2-2020.2 [19]. Sequence reads were denoised using DADA2 and truncated at $220 \mathrm{bp}$ and $120 \mathrm{bp}$ for forward and reverse reads, respectively, aligned, filtered, checked for chimeras, and OTUs were classified based on the Greengenes classifier, 13_8 99\% OTUs from 515F/806R region of sequences (gg-13-8-99-515-806-nb) [20,21]. Functional predictions were made analyzing sequences by PICRUSt2 to infer functional content and visualized using STAMP v2.1.3 $[22,23]$.

\subsection{Statistical Analyses}

Data were evaluated ANOVA, PERMANOVA, regression analysis, or multivariate analysis techniques. The Benjamini-Hochberg method was used to adjust $p$-values to control the false discovery rate. Data analyses were conducted using Systat, version 13.0 (Systat Software, Inc., San Jose, CA, USA), CLC Genomics Workbench version 20.0.4 (Qiagen Bioinformatics, Redwood City, CA, USA) and RStudio version 1.1.456 (RStudio, Boston, MA, USA) running R version 3.6.3 (The $\mathrm{R}$ Foundation for Statistical Computing, Vienna, Austria).

\section{Results and Discussion}

\subsection{Development of a Mammary Cancer Screening Model}

The PyMT ongene, driven by the MMTV promoter, is recognized to be an aggressive transgenic model for breast cancer that rapidly invades and metastasizes [12,13]. A time series documenting the pathogenesis of the disease process has been reported [24]. However, to our knowledge, there has not been a detailed analysis of pathology development during the first four weeks post weaning. Using an image analysis system that was designed to quantify mammary pathology development in whole mount preparations of the mammary gland [15] (Figure 1A), it is clear that there is a linear increase in lesion size over the timeframe of 0 to 4 weeks post weaning (Figure 1B). This pattern of cell growth is analogous to the increase in cell number observed in cell culture models, which occurs over a period of 3 to 7 days depending on the cell line. The mammary pathologies were evaluated histopathologically and were diagnosed as mammary carcinoma. 
A

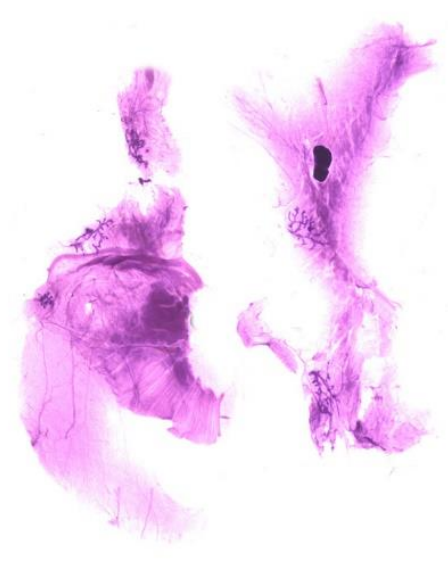

C

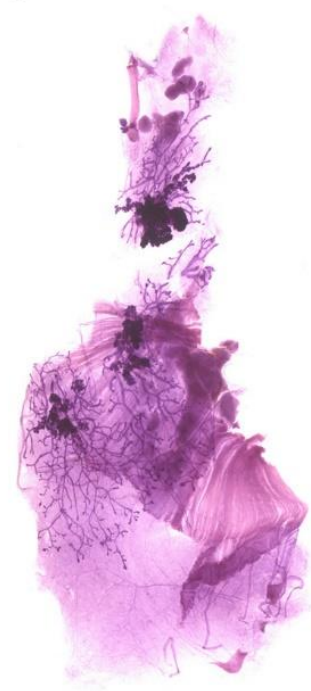

\section{B}

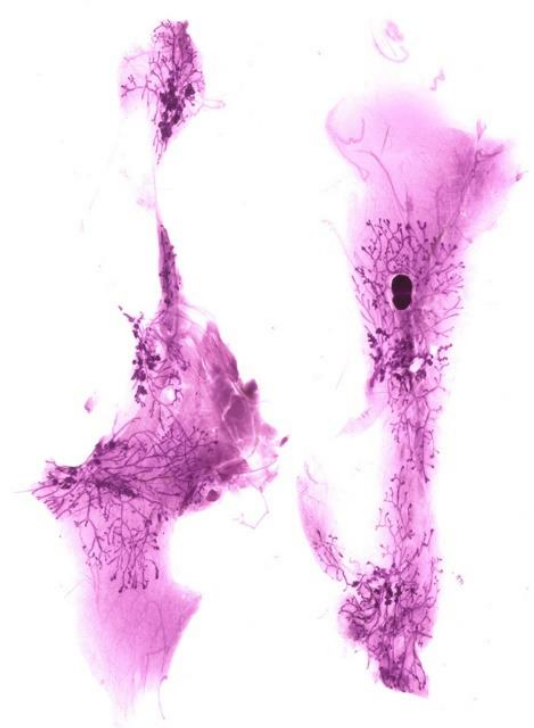

D

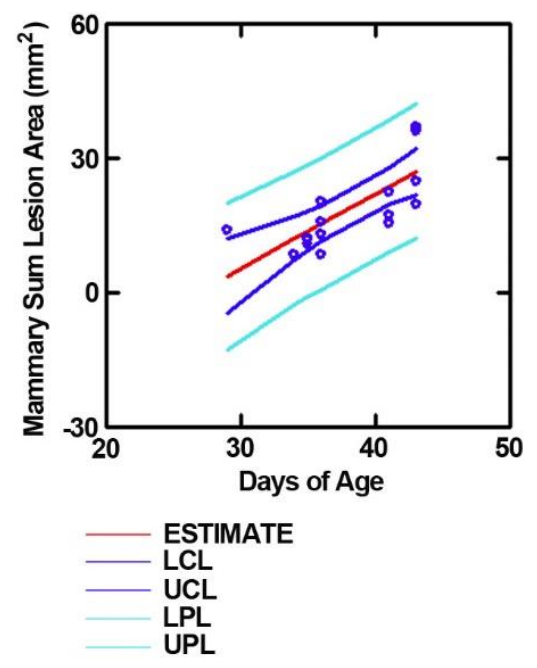

Figure 1. Representative mammary glands wholemounts from PyMT (pP) female mice over a 22 day time course. Panel (A), 21 DOA; panel (B), 36 DOA; panel (C) 43 DOA; panel (D) is the linear regression for increase in mammary pathology area over time, $p<0.001$.

\subsection{Effect of Common Bean on the Expansion of Mammary Pathologies In Vivo}

As shown in Figure 1A, there is a limited amount of mammary epithelium primarily localized to the region directly around each teat when hemizygous PyMT female mice are 21 days of age. Feeding common bean was initiated at this time and continued for two weeks. As shown in Table 1, common bean suppressed the expanison of the mammary pathologies in each mammary fat pad. Figure 2 shows representative mammary gland whole mounts illustrating the nature of the differences that exist between control and bean fed mice 14 days after randomization to either control or bean formulated diets. 
Table 1. Effect of common bean on mammary pathology expansion.

\begin{tabular}{cccc}
\hline Sum Lesion Area $\left(\mathbf{m m}^{2}\right)$ & CTRL $(\boldsymbol{n}=\mathbf{9})$ & Bean $(\boldsymbol{n}=\mathbf{1 0})$ & $\boldsymbol{p}$-Value \\
\hline Mammary gland 1 & $7.64 \pm 1.00$ & $4.00 \pm 0.66$ & 0.007 \\
Mammary gland 2 & $3.37 \pm 0.74$ & $1.80 \pm 0.54$ & 0.092 \\
Mammary gland 3 & $5.38 \pm 1.10$ & $2.26 \pm 0.45$ & 0.014 \\
Mammary gland 4 & $6.00 \pm 2.06$ & $1.66 \pm 0.33$ & 0.043 \\
Mammary gland 5 & $5.86 \pm 1.79$ & $1.95 \pm 0.29$ & 0.028 \\
\hline
\end{tabular}

Values are means \pm SEM; CTRL $n=9$, Bean $n=10$.

A

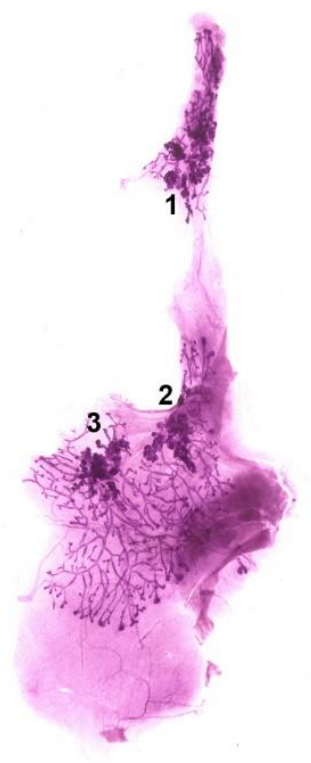

B

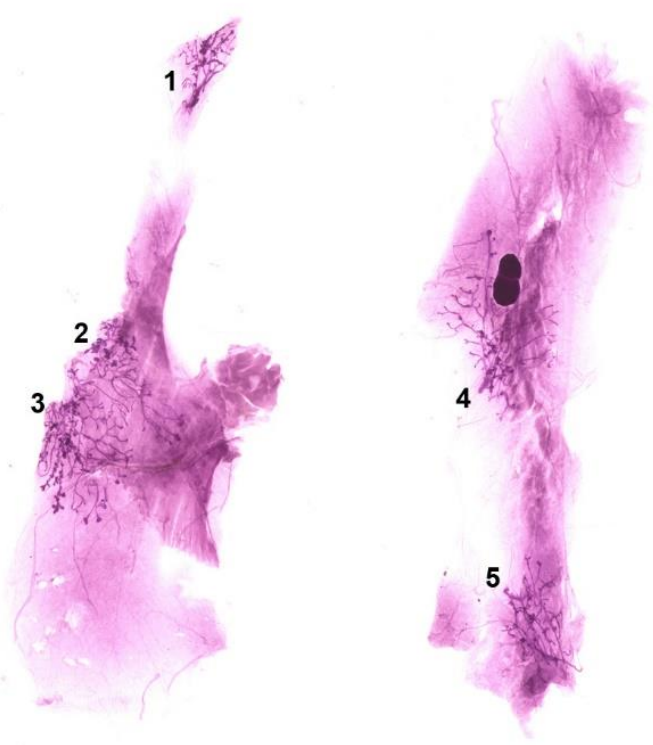

Figure 2. Representative mammary gland whole mounts from PyMT female mice fed control or bean containing purified diet for two weeks. (A) Control diet; (B) Bean diet; Location of mammary gland nipples 1-5 are labeled in each panel.

\subsection{Effect of Common Bean on Cell Signaling Pathways in Mammary Pathologies In Vivo}

The experiment reported in Table 1 was repeated, but the size of mammary pathologies was assessed on the mammary gland chains excised from the right side of each mouse. The total area of mammary pathology was reduced by feeding common bean (Figure 3A). The glands on the left side of each mouse were removed and snap frozen in liquid nitrogen. Subsequently, protein was extracted and subjected to nano immuno-capillary electrophoresis on a Simple WES system. Our previously published work has shown that AMP activated protein kinase (AMPK) is activated in the mammary gland and tumors of bean fed animals [10]. That observation was confirmed in this model system (Figure 2B). 


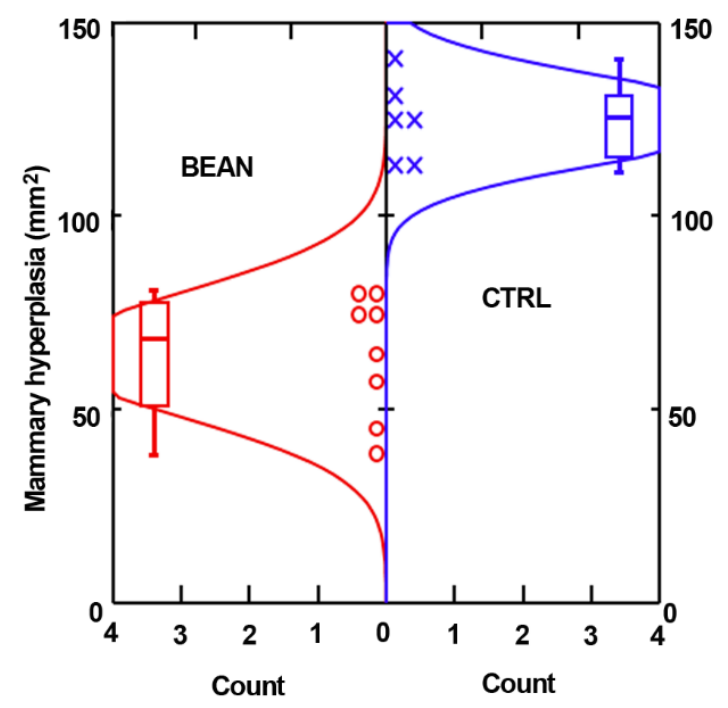

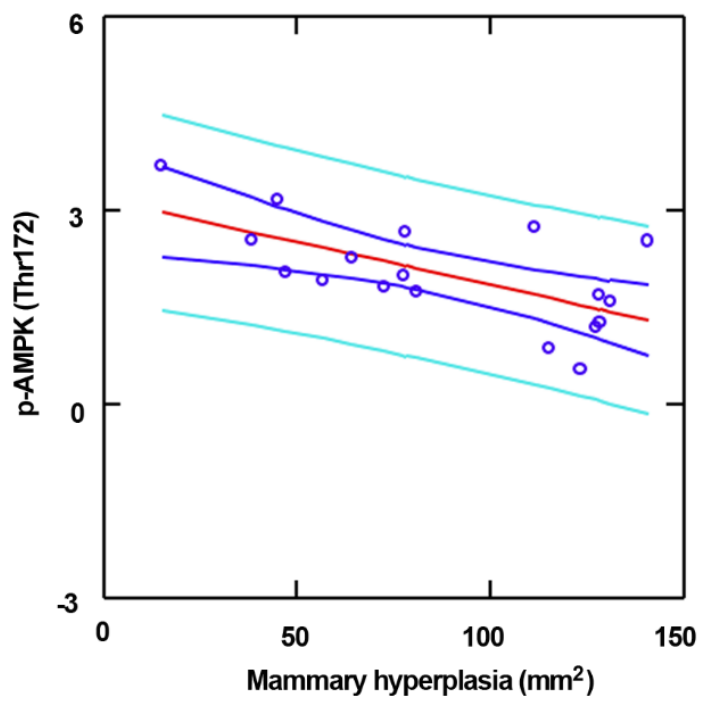

Figure 3. Effect of bean feeding on PyMT driven mammary carcinogenesis, two sample t-test $p<0.01$.

(A) Mammary pathology expansion, $p<0.01$; (B) Levels of phospho AMPK mammary pathologies.

A total of 7 proteins, identified on Figure 4, were measured by WES analysis. Those data were evaluated using Ingenuity Pathway Analysis (Qiagen, Redwood City, CA, USA), and the Endocannabinoid Cancer Inhibition Pathway was identified as being highly enriched in its activation $\left(p<1.0 \times 10^{-7}\right.$, Figure 4) [25]. Identification of the potential involvement of this pathway was unanticipated and illustrates the value of data driven investigation that the screening model provides.

The expression of the many genes/proteins shown in Figure 4 requires further investigation. However, an equally important objective is the determination of potential mediators of activation. Since the low molecular weight fraction of bean had no effect on human breast cancer cell lines grown in culture yet, cannabinoids have been reported to inhibit the growth of breast cell lines in vitro $[26,27]$, we decided to investigate an alternative source of mediation, the gut associated microbiome. This approach was judged to have merit for two reasons. First, we have reported that common bean has a high content of dietary fiber, a fact that is commonly overlooked [28-30]. Dietary fiber is recognized inducers of effects on the gut microbiome. Second, there is an emerging literature that shows a mechanistic relationships between the gut associated microbiome and endocannabinoid signaling, potential via the alteration of gut microbial ecology such that microbial species are favored that express gene involved in the synthesis of bioactive lipids [31,32]. Recognized endogenously synthesized lipid that binds cannabinoid receptors include 2-arachidonoyl glycerol and arachidonoyl ethanolamide (anandamide) [33]. The endocannabinoids inhibit cancer cell proliferation, arrest the cell cycle, induce cell death, mainly through apoptosis and autophagy, prevent tumor spread, and block angiogenesis in the tumor microenvironment. 


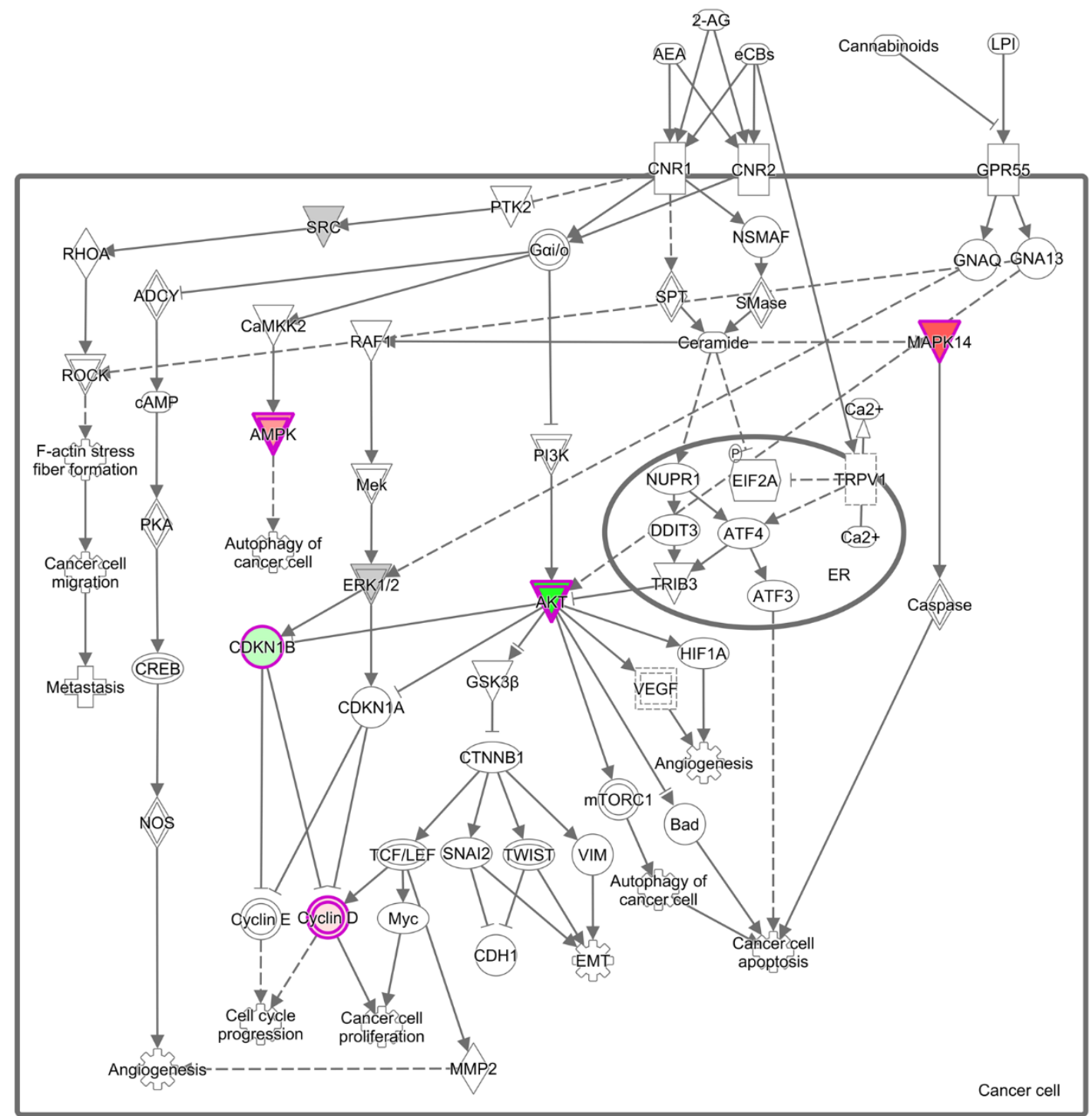

Figure 4. Endocannabinoid Cancer Inhibition Pathway. Proteins that were assessed and that are nodes in this pathway are circumscribed in red. Pink shape-fill indicates activation and green shape-fill indicates inactivation.

\subsection{Effect of Common Bean on the Gut Associated Microbiome}

As noted above, the finding that the endocannabinoid signaling pathway was implicated in the mediation of the effects of common bean was not anticipated. Although we have isolated the content of various segments of the intestinal tract for microbiome analyses in other studies [14,34], intestinal content was not isolated in the screening study described in Section 3.2. Rather, as a test-of-biological plausibility, DNA from the cecal content from the bean feeding study reported in [14] was subjected to library prep, 16s rRNA sequencing, and bioinformatic analyses.

As shown in Table 2, there was a marked increase in bacteria classified in the phylum Bacteriodetes and a commensurate reduction in the bacteria in the phylum Firmicutes in mice fed the bean versus control diet, both effects FDR adjusted $p$-value $<0.001$. Other statistically significant differences were also noted. We next assessed the impact of bean consumption through the lens of phylogenetic diversity and as shown in Figure 5. Diversity was reduced by bean feeding suggesting that bean feeding created a niche environment that great numbers of fewer types of microbes. If bean as a food is considered from the food pharmacological perspective, this prebiotic effect is what would be expected from a food intervention targeting the gut microbiome. 
Table 2. Effect of bean feeding on microbial relative abundance at the level of phylum.

\begin{tabular}{cccccc}
\hline Phylum & log2FC & $p \_$Value & FDR & Ctrl_Log2_Mean & Bean_Log2_Mean \\
\hline (Unknown Phylum) Bacteria & -0.051354766 & 0.038652403 & 0.064420671 & -6.581462293 & -6.632817059 \\
Actinobacteria & -0.142413323 & 0.016577252 & 0.033154504 & -6.501442867 & -6.64385619 \\
Bacteria & -0.094320142 & 0.064927787 & 0.081159733 & -6.483667922 & -6.577988064 \\
Bacteroidetes & 1.784902055 & 0.000195928 & 0.000979641 & -2.165674619 & -0.380772565 \\
Candidatus Saccharibacteria & -0.004295945 & 0.373900966 & 0.373900966 & -6.639560244 & -6.64385619 \\
Deferribacteres & -1.418705665 & 0.011339537 & 0.028348842 & -5.066253673 & -6.484959337 \\
Firmicutes & -1.759348332 & 0.000525378 & 0.001751259 & -0.536789118 & -2.296137451 \\
Proteobacteria & -1.94107296 & $5.69 \mathrm{E}-05$ & 0.000568878 & -3.558157983 & -5.499230943 \\
Tenericutes & -0.007939984 & 0.373900966 & 0.373900966 & -6.635916206 & -6.64385619 \\
Verrucomicrobia & 1.403597718 & 0.059447941 & 0.081159733 & -6.64385619 & -5.240258472 \\
\hline
\end{tabular}

Log2 fold change: Log2FC; false discovery rate: FDR.

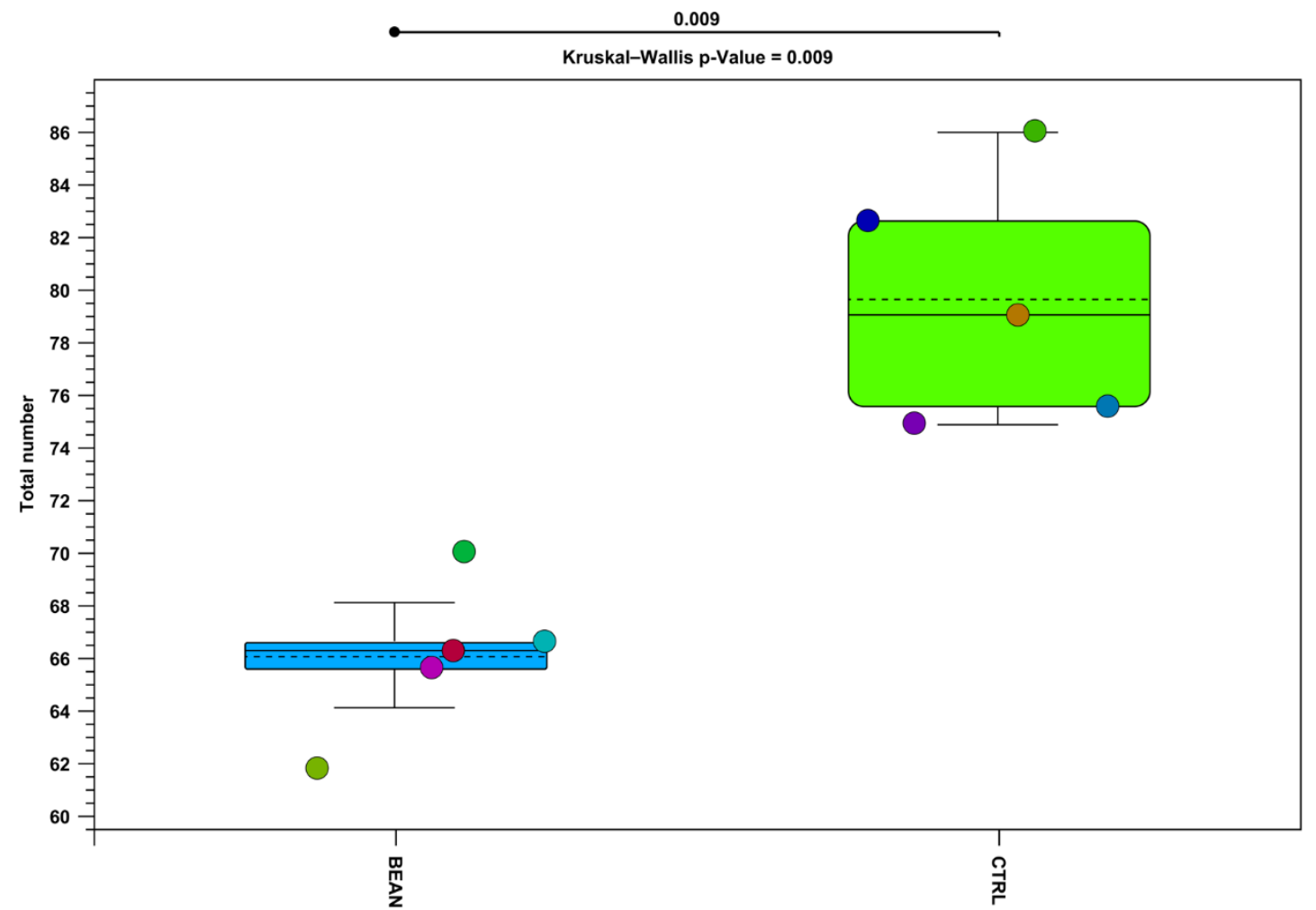

Figure 5. Effect of bean feeding on phylogenetic diversity. Alpha diversity, $p=0.009$ BEAN $n=5$, CTRL $n=5$.

The effect of bean feeding on beta diversity was also assessed (Figure 6). There was complete separation between mice consuming the control diet versus the bean diet formulation. The first principle componet accounted for $81 \%$ of the variability in the mathematical model. This finding is consistent with bean feeding establishing a distinct ecological environment with the gut that favors distinctly different microbial populations (amount and type) than the control diet. This is most likely due, at least in part to the dietary fiber component of the carbohydrates present in common bean that escape digestion and absorption in the small intestine. It is also possible that some protein present in common bean is not completely digested by the time it leaves the small intestine and protein would foster colony forming by distinct populations of microorganisms. 


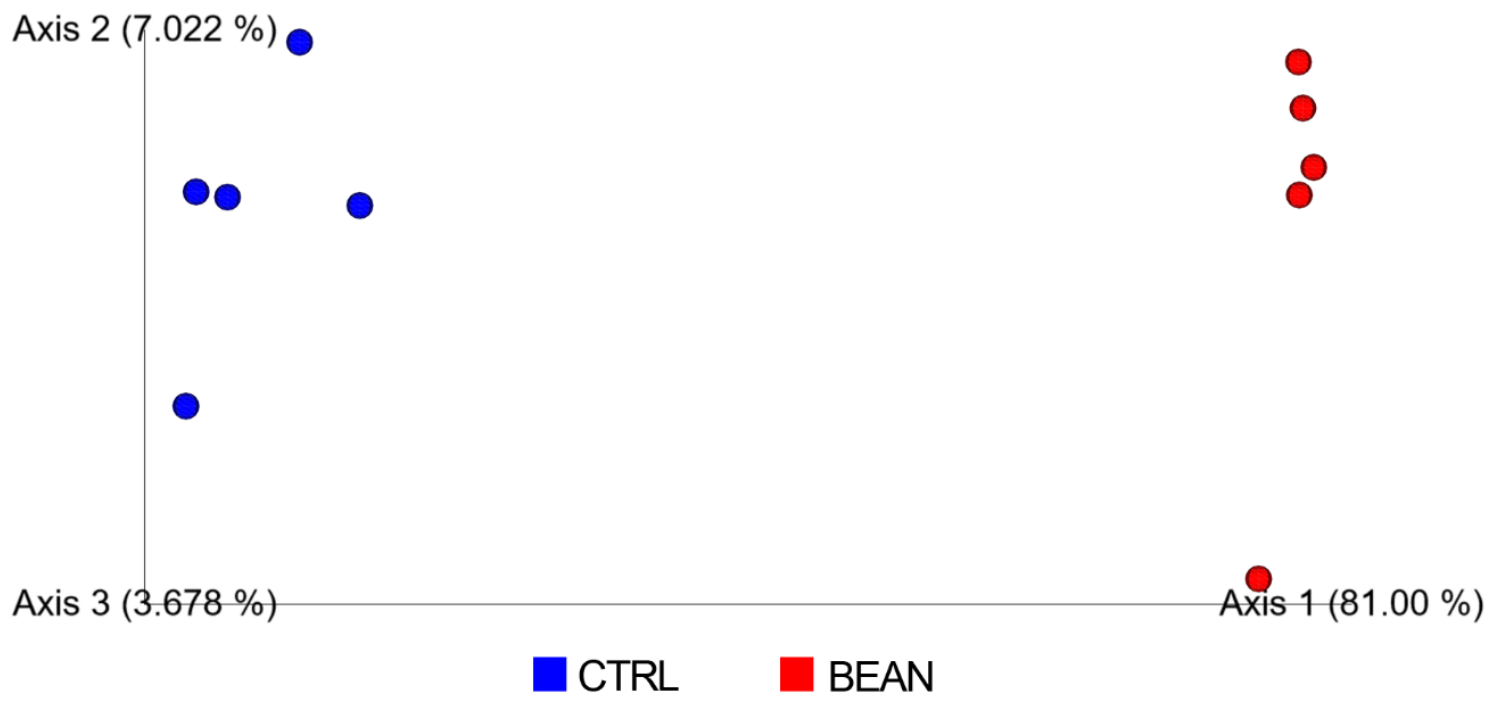

Figure 6. Effect of bean on beta diversity within the gut.

The data from the microbial analyses was also evaluated to predict functional differences in the microbiomes of control and bean fed mice using PICRUSt with statistical analyses in STAMP. As this was a test for biological plausibility that bean mediates effects via enrichment of the synthesis of bioactive lipids, the presentation of results was to genes in metabolic pathways involving fatty acid oxidation and phospholipid synthesis, fatty acid synthesis, and fatty acid elongation. The findings predict that microbial fatty acid oxidation and phosphlipid biosynthesis are predicted too be suppressed in bean fed mice; whereas, fatty acid synthesis was unchanged and fatty acid elongation markedly enhanced. These changes are consistent with a capacity for enhanced synthesis of long chain fatty acids, potentially bioactive lipids, in bean fed mice. However, at this point, only biological plausibility is apparent. The key next steps in developing a testable hypothesis include: (1) the evaluation of the metatranscriptome, since Figure 7 is only a summary of predicted functions, (2) the evaluation of the metabolome present in the gut luminal content and the plasma metabolome of the host, and (3) the assessment of the activity of the cannabinoid signaling pathway in the luminal epithelial of various segments of the intestinal tract. 
A

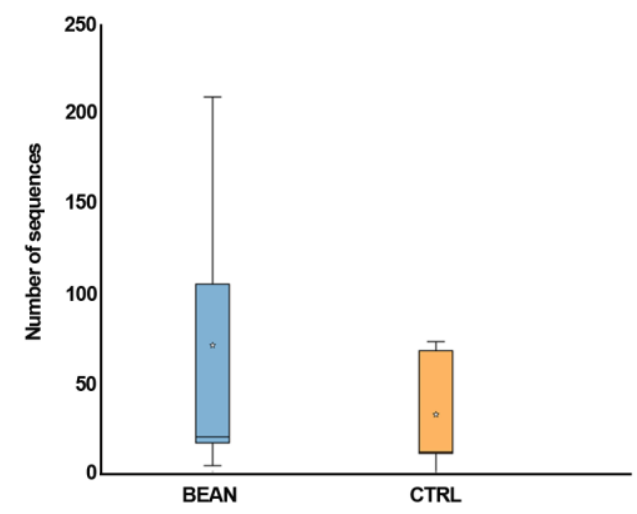

C

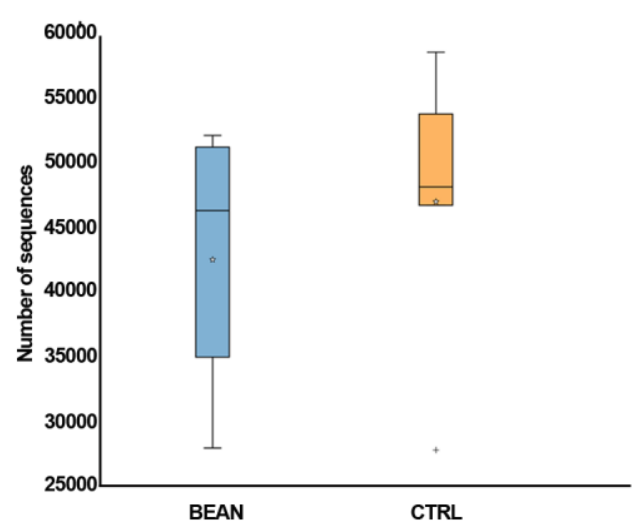

B

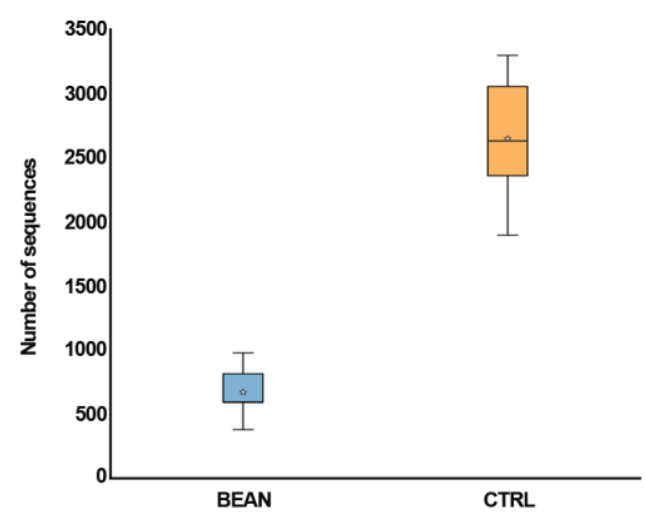

D

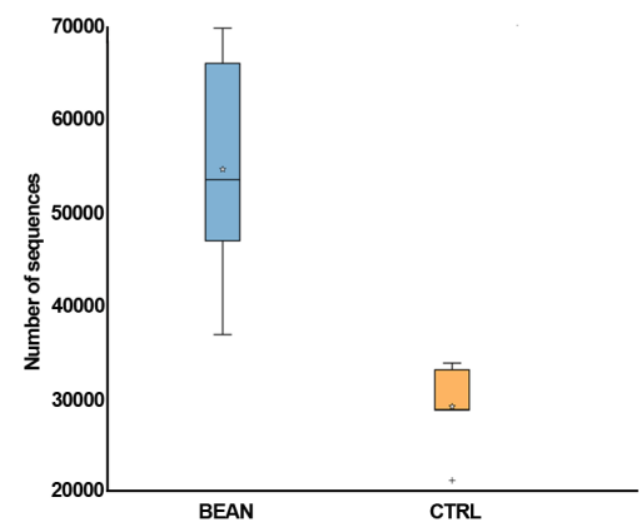

Figure 7. Predicted functional differences of cecal microbial populations in control and bean fed mice. (A) FASYN initial pathway, $p=0.424$; (B) FAO pathway, $p=1.69 \times 10^{-4}$; (C) PHOSLIPSYN pathway, $p$ $=2.14 \times 10^{-5}$; (D) FASYN-ELONG pathway, $p=9.92 \times 10^{-6}$; CTRL $n=5$, BEAN $n=5$.

\section{Concluding Comments}

This test of concept investigation reports a short-term in vivo screening assay for effects on breast cancer cell growth that permits the evaluation of foods alone or in combination. Moreover, we demonstrate the feasibility of assessing changes in cell signaling in the target tissue, i.e., the breast as well as in a potential source from which mediation of effects on cancer may be derived, i.e., the gut associated microbiome. We do not envision this particularly version of the screening model to be without the opportunity for improvement and optimization for various questions of interest, but we do see it as opening the door to rapid assessment of foods for anticancer activity and for the identification of the mechanisms by which protective effects are mediated.

Author Contributions: Conceptualization, H.J.T.; methodology, E.S.N., J.N.M., T.L.W.; formal analysis, E.S.N., J.N.M., H.J.T.; investigation, H.J.T.; data curation, E.S.N., J.N.M., H.J.T.; writing-original draft preparation, H.J.T.; writing - review and editing, E.S.N., J.N.M., H.J.T., T.L.W.; supervision, E.S.N., J.N.M., H.J.T.; project administration, H.J.T.; funding acquisition, H.J.T.

Funding: This research was funded in part by the Agricultural Research Service, U.S. Department of Agriculture, grant number 3060-21650-001-09S.

Acknowledgments: The authors thank Barry Ogg and Vanessa Fitzgerald for technical assistance and Meenakshi Sing for histopathological classification of mammary pathologies.

Conflicts of Interest: The authors declare no conflict of interest. The funders had no role in the design of the study; in the collection, analyses, or interpretation of data; in the writing of the manuscript, or in the decision to publish the results. 


\section{References}

1. NIH. The Science behind Healthy Eating Patterns; 2020.

2. NIH. 2020-2030 Strategic Plan for NIH Nutrition Research. 2020. Available online: https://www.niddk.nih.gov/about-niddk/strategic-plans-reports/strategic-plan-nih-nutrition-research (accessed on 15 October 2020).

3. Frame, L.A.; Costa, E.; Jackson, S.A. Current Explorations of Nutrition and the Gut Microbiome: A Comprehensive Evaluation of the Review Literature. Nutr. Rev. 2020, doi:10.1093/nutrit/nuz106.

4. McBurney, M.I.; Davis, C.; Fraser, C.M.; Schneeman, B.O.; Huttenhower, C.; Verbeke, K.; Walter, J.; Latulippe, M.E. Establishing What Constitutes a Healthy Human Gut Microbiome: State of the Science, Regulatory Considerations, and Future Directions. J. Nutr. 2019, 149, 1882-1895, doi:10.1093/jn/nxz154.

5. Bilotta, A.J.; Cong, Y. Gut microbiota metabolite regulation of host defenses at mucosal surfaces: Implication in precision medicine. Precis. Clin. Med. 2019, 2, 110-119, doi:10.1093/pcmedi/pbz008.

6. Rodgers, G.P.; Collins, F.S. Precision Nutrition - The Answer to "What to Eat to Stay Healthy". JAMA 2020, doi:10.1001/jama.2020.13601.

7. Adebamowo, C.A.; Cho, E.; Sampson, L.; Katan, M.B.; Spiegelman, D.; Willett, W.C.; Holmes, M.D. Dietary flavonols and flavonol-rich foods intake and the risk of breast cancer. Int. J. Cancer 2005, 114, 628-633.

8. Murtaugh, M.A.; Sweeney, C.; Giuliano, A.R.; Herrick, J.S.; Hines, L.; Byers, T.; Baumgartner, K.B.; Slattery, M.L. Diet patterns and breast cancer risk in Hispanic and non-Hispanic white women: The Four-Corners Breast Cancer Study. Am. J. Clin. Nutr. 2008, 87, 978-984, doi:10.1093/ajcn/87.4.978.

9. Thompson, M.D.; Mensack, M.M.; Jiang, W.; Zhu, Z.; Lewis, M.R.; McGinley, J.N.; Brick, M.A.; Thompson, H.J. Cell signaling pathways associated with a reduction in mammary cancer burden by dietary common bean (Phaseolus vulgaris L.). Carcinogenesis 2012, 33, 226-232, doi:10.1093/carcin/bgr247.

10. Thompson, M.D.; Thompson, H.J.; Brick, M.A.; McGinley, J.N.; Jiang, W.; Zhu, Z.; Wolfe, P. Mechanisms associated with dose-dependent inhibition of rat mammary carcinogenesis by dry bean (Phaseolus vulgaris, L.). J. Nutr. 2008, 138, 2091-2097, doi:10.3945/jn.108.094557.

11. Mensack, M.M.; Fitzgerald, V.K.; Lewis, M.R.; Thompson, H.J. Characterization of low molecular weight chemical fractions of dry bean (Phaseolus vulgaris) for bioactivity using Caenorhabditis elegans longevity and metabolite fingerprinting. J. Agric. Food Chem. 2010, 58, 6697-6705, doi:10.1021/jf1007538.

12. Davie, S.A.; Maglione, J.E.; Manner, C.K.; Young, D.; Cardiff, R.D.; MacLeod, C.L.; Ellies, L.G. Effects of $\mathrm{FVB} / \mathrm{NJ}$ and C57B1/6J strain backgrounds on mammary tumor phenotype in inducible nitric oxide synthase deficient mice. Transgenic Res. 2007, 16, 193-201, doi:10.1007/s11248-006-9056-9.

13. Guy, C.T.; Cardiff, R.D.; Muller, W.J. Induction of mammary tumors by expression of polyomavirus middle $\mathrm{T}$ oncogene: A transgenic mouse model for metastatic disease. Mol. Cell. Biol. 1992, 12, 954-961, doi:10.1128/mcb.12.3.954.

14. Neil, E.S.; McGinley, J.N.; Fitzgerald, V.K.; Lauck, C.A.; Tabke, J.A.; Streeter-McDonald, M.R.; Yao, L.; Broeckling, C.D.; Weir, T.L.; Foster, M.T.; et al. White Kidney Bean (Phaseolus vulgaris L.) Consumption Reduces Fat Accumulation in a Polygenic Mouse Model of Obesity. Nutrients 2019, 11, 2780, doi:10.3390/nu11112780.

15. McGinley, J.N.; Thompson, H.J. Quantitative assessment of mammary gland density in rodents using digital image analysis. Biol. Proced. Online 2011, 13, 4, doi:10.1186/1480-9222-13-4.

16. Singh, M.; McGinley, J.N.; Thompson, H.J. A comparison of the histopathology of premalignant and malignant mammary gland lesions induced in sexually immature rats with those occurring in the human. Lab. Investig. 2000, 80, 221-231.

17. Thompson, H.J.; McGinley, J.N.; Neil, E.S.; Brick, M.A. Beneficial Effects of Common Bean on Adiposity and Lipid Metabolism. Nutrients 2017, 9, 998, doi:10.3390/nu9090998.

18. Kozich, J.; Schloss, P.; Baxter, N.; Jenior, M.; Koumpouras, C.; Bishop, L. 16S rRNA Sequencing with the Illumina MiSeq: Library Generation, QC, \& Sequencing. Available online: https://github.com/SchlossLab/MiSeq_WetLab_SOP (accessed on 12 August 2019).

19. Bolyen, E.; Rideout, J.R.; Dillon, M.R.; Bokulich, N.A.; Abnet, C.C.; Al-Ghalith, G.A.; Alexander, H.; Alm, E.J.; Arumugam, M.; Asnicar, F.; et al. Reproducible, interactive, scalable and extensible microbiome data science using QIIME 2. Nat. Biotechnol. 2019, 37, 852-857, doi:10.1038/s41587-019-0209-9.

20. Callahan, B.J.; McMurdie, P.J.; Rosen, M.J.; Han, A.W.; Johnson, A.J.A.; Holmes, S.P. DADA2: Highresolution sample inference from Illumina amplicon data. Nat. Methods 2016, 13, 581-583, doi:10.1038/nmeth.3869. 
21. McDonald, D.; Price, M.N.; Goodrich, J.; Nawrocki, E.P.; DeSantis, T.Z.; Probst, A.; Andersen, G.L.; Knight, R.; Hugenholtz, P. An improved Greengenes taxonomy with explicit ranks for ecological and evolutionary analyses of bacteria and archaea. ISME J. 2012, 6, 610-618, doi:10.1038/ismej.2011.139.

22. Douglas, G.M.; Maffei, V.J.; Zaneveld, J.; Yurgel, S.N.; Brown, J.R.; Taylor, C.M.; Huttenhower, C.; Langille, M.G.I. PICRUSt2: An improved and extensible approach for metagenome inference. bioRxiv 2019, 672295, doi:10.1101/672295.

23. Parks, D.H.; Tyson, G.W.; Hugenholtz, P.; Beiko, R.G. STAMP: Statistical analysis of taxonomic and functional profiles. Bioinformatics 2014, 30, 3123-3124, doi:10.1093/bioinformatics/btu494.

24. Lin, E.Y.; Pollard, J.W. Macrophages: Modulators of breast cancer progression. Novartis Found. Symp. 2004, 256, 158-168; discussion 168-172, 259-169.

25. Kovalchuk, O.; Kovalchuk, I. Cannabinoids as anticancer therapeutic agents. Cell Cycle 2020, 19, 961-989, doi:10.1080/15384101.2020.1742952.

26. Takeda, S.; Okajima, S.; Miyoshi, H.; Yoshida, K.; Okamoto, Y.; Okada, T.; Amamoto, T.; Watanabe, K.; Omiecinski, C.J.; Aramaki, H. Cannabidiolic acid, a major cannabinoid in fiber-type cannabis, is an inhibitor of MDA-MB-231 breast cancer cell migration. Toxicol. Lett. 2012, 214, 314-319, doi:10.1016/j.toxlet.2012.08.029.

27. Hermanson, D.J.; Marnett, L.J. Cannabinoids, endocannabinoids, and cancer. Cancer Metastasis Rev. 2011, 30, 599-612, doi:10.1007/s10555-011-9318-8.

28. Chen, Y.; McGee, R.; Vandemark, G.; Brick, M.; Thompson, H.J. Dietary Fiber Analysis of Four Pulses Using AOAC 2011.25: Implications for Human Health. Nutrients 2016, 8, 829, doi:10.3390/nu8120829.

29. Thompson, H.J.; Brick, M.A. Perspective: Closing the Dietary Fiber Gap: An Ancient Solution for a 21st Century Problem. Adv. Nutr. 2016, 7, 623-626, doi:10.3945/an.115.009696.

30. Kleintop, A.E.; Echeverria, D.; Brick, L.A.; Thompson, H.J.; Brick, M.A. Adaptation of the AOAC 2011.25 integrated total dietary fiber assay to determine the dietary fiber and oligosaccharide content of dry edible beans. J. Agric. Food Chem. 2013, 61, 9719-9726, doi:10.1021/jf403018k.

31. Muccioli, G.G.; Naslain, D.; Bäckhed, F.; Reigstad, C.S.; Lambert, D.M.; Delzenne, N.M.; Cani, P.D. The endocannabinoid system links gut microbiota to adipogenesis. Mol. Syst. Biol. 2010, 6, 392, doi:10.1038/msb.2010.46.

32. Manca, C.; Boubertakh, B.; Leblanc, N.; Deschenes, T.; Lacroix, S.; Martin, C.; Houde, A.; Veilleux, A.; Flamand, N.; Muccioli, G.G.; et al. Germ-free mice exhibit profound gut microbiota-dependent alterations of intestinal endocannabinoidome signaling. J. Lipid Res. 2020, 61, 70-85, doi:10.1194/jlr.RA119000424.

33. Lu, H.-C.; Mackie, K. An Introduction to the Endogenous Cannabinoid System. Biol. Psychiatry 2016, 79, 516-525, doi:10.1016/j.biopsych.2015.07.028.

34. McGinley, J.N.; Fitzgerald, V.K.; Neil, E.S.; Omerigic, H.M.; Heuberger, A.L.; Weir, T.L.; McGee, R.; Vandemark, G.; Thompson, H.J. Pulse Crop Effects on Gut Microbial Populations, Intestinal Function, and Adiposity in a Mouse Model of Diet-Induced Obesity. Nutrients 2020, 12, 593, doi:10.3390/nu12030593.

Publisher's Note: MDPI stays neutral with regard to jurisdictional claims in published maps and institutional affiliations.

(C) 2020 by the authors. Submitted for possible open access publication under the terms and conditions of the Creative Commons Attribution (CC BY) license (http://creativecommons.org/licenses/by/4.0/). 